\title{
Infrared Image Detail Enhancement Approach Based on Improved Joint Bilateral filter
}

\author{
Ning Liu*,1 Xiaohong Chen ${ }^{1}$ \\ 1. Nanjing University of Posts and Telecommunications, School of Optoelectronics \\ Engineering, 9 Wenyuan Ave. Nanjing, Jiangsu Province, 210023. \\ *Corresponding author: coolboy006@sohu.com
}

\begin{abstract}
In this paper, we proposed a new infrared image detail enhancement approach. This approach could not only achieve the goal of enhancing the digital detail, but also make the processed image much closer to the real situation. Inspired by the joint-bilateral filter, two adjacent images were utilized to calculate the kernel functions in order to distinguish the detail information from the raw image. We also designed a new kernel function to modify the joint-bilateral filter and to eliminate the gradient reversal artifacts caused by the non-linear filtering. The new kernel is based on an adaptive emerge coefficient to realize the detail layer determination. The detail information was modified by the adaptive emerge coefficient along with two key parameters to realize the detail enhancement. Finally, we combined the processed detail layer with the base layer and rearrange the high dynamic image into monitor-suited low dynamic range to achieve better visual effect. Numerical calculation showed that this new technology has the best value compare to the previous research in detail enhancement. Figures and data flowcharts were demonstrated in the paper.
\end{abstract}

Keywords: infrared images; joint bilateral filter; digital detail enhancement; high dynamic compression

\section{Introduction}

Infrared imaging has been applied in various fields for both military and civilian use, such as design, test, manufacturing, chemical imaging, night vision, surveillance in security, target signature measurement, tracking and so on $[1,2,9,10,11,12]$. Normally the infrared cameras have a wide dynamic range more than 14-bit. The usual way to display the infrared images on a screen is to compress them with a histogram equalization algorithm. However this method cannot fully show the entirely detail information. In recent years, many approaches have been widely investigated with a number of detail enhancement techniques proposed in literature $[3,4,13,14,15,16]$. All these methods aim to enhance the quality of an infrared image and reproduce the reality of the captured scene or target as possible as they could.

There are many classic edge extraction operators for enhancing an image. These operators make the edge sharper or the details of an image extruder, such as Sobel $[5,6,7]$, Prewitt [17,18], Log [19,20], Laplacian [20] and so on. However the results were not satisfied. The real problem for the researchers is that how we can fully spot out the very tiny fluctuation of the gray value of an infrared image. However, the traditional methods can only simply watch the approximate morphology of an image, but not the total content in it. Many researchers focused on figuring out the solution. For example, in 2011, Zuo et al. [8] proposed a novel method of reality reproduction for high-dynamic-range infrared images called BF\&DDE, which worked better in detail enhancement and noise reduction than other methods. This approach used a modified bilateral filter to separate a raw image into detail 
layer and base layer. The base layer is histogram equalized to fit in the display range, while the detail layer is added back after an adaptive gain control process. In 2014, we proposed a method to achieve this goal called the GIF\&DDE [23] in which a guided image filter was used to separate the raw image. Both these two methods could greatly increase the image quality and enhance the image into a whole new level for visual observation. However these two methods have certain drawbacks. For example, the generic problem exists in these two methods are the reversal artifacts. When the image has too many strong edges in it, both these methods cannot fully eliminate them. In GIF\&DDE, this could happen because the principle of the filter itself. Since GIF is a linear filter, it is surely hard to deal with high gradient information within the edges. In BF\&DDE, Zuo[8] uses adaptive Gaussian filter to suppress the gradient reversal effect with a big filter window, if the edges are too strong, the size of the filter window usually bigger than $15 \times 15$, which could be time-consuming. In our further research, we find that these two methods still can be improved in enhancing the digital details within the image scene. Thus, we have figured out a way to do so in this paper. This method is inspired by the joint-bilateral filter [24] which is usually used in color image process. We use two adjacent infrared images in the video sequence to calculate the detail layer and get a better detail content. We also design a novel kernel function to calculate with the joint-bilateral filter during the processing procedure. This kernel function is sensitive to the gradient structure within the image, which could be given better elimination of the reversal artifacts. The proposed method can be more flexible of reducing the background noises. This method can achieve the greater effect of detail enhancement and noise reduction, and furthermore, works better near strong edges.

The paper is organized as follows: in section 2, the detailed mechanism of the proposed method is introduced; in section 3, the experimental results are introduced; in section 4, we give a conclusion of our method.

\section{2 the mechanism of the proposed algorithm}

\section{1 the principle of the joint-bilateral filter}

J. Kopf et al. [24] proposed the joint-bilateral filter (JBF) in 2007, which was first used in processing the colored images. They used the high resolution colored image as the reference image, and used the JBF to up-sample the low resolution image in order to improve the resolution. In our research, we find this procedure is also very effective when dealing with the infrared images. The basic difference is that, we do not up-sample the original image, but choose two adjacent infrared images as a pair, and use the first image as the basic image while the second one as the reference image. We use the JBF to calculate the kernel function inside the two images, and then applied the kernel on the reference image to get the filtered result. The filtered result could be treated as the base layer of the basic image, which is used to determine the detail layer by a simple subtraction with the reference image. We also design an effective filter coefficient to deal with the gradient reversal artifacts which happens in using the non-linear filter to process the original image $[9,10]$. This approach is proved better and faster than the Gaussian filter mentioned in the BF\&DDE [8]. Finally, we combine the processed detail layer and the base layer back together to achieve the goal of detail enhancement of the infrared image. Fig.1 gives an illustration of the mechanism of the proposed algorithm. 


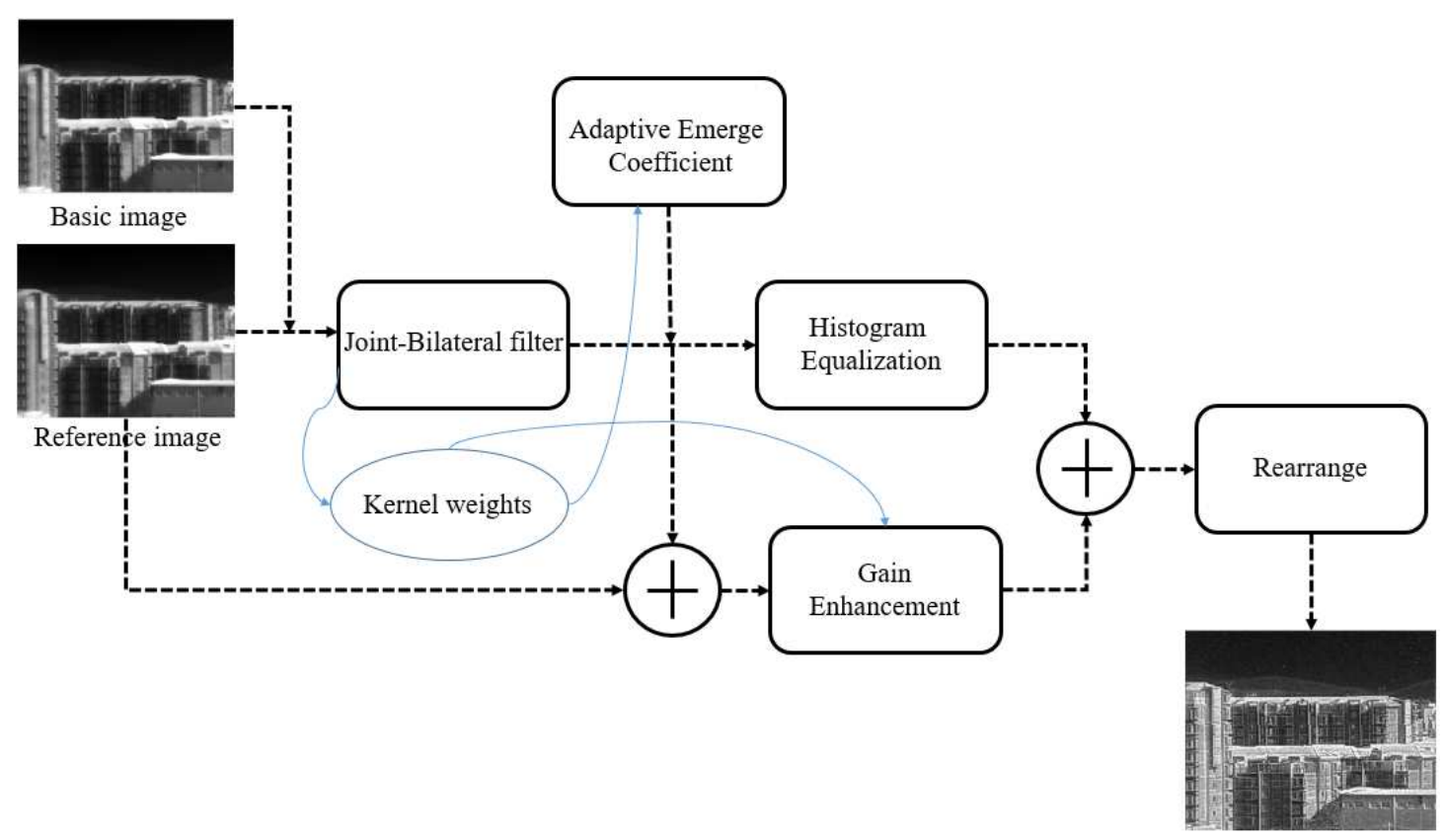

Fig. 1 the scheme of the proposed algorithm. Blue arrows indicates that these modules are controlled or can be adjusted by the kernel weights.

The expression of joint-bilateral filter is given as eq.1. The primary difference between the joint-bilateral filter and the traditional bilateral filter is that the JBF uses two adjacent images as the input, and then calculate the kernel weights matrix [24].

$$
I_{J B F}=k \sum_{i^{\prime}, j^{\prime} \in \Omega} \omega_{S}\left(\left\|i-i^{\prime}, j-j^{\prime}\right\|\right) \omega_{r}\left(\left\|I_{B}-I_{R}\right\|\right) I_{R}
$$

Where the $I_{J B F}$ is the JBF filtered result, $I_{R}$ is the reference image, $I_{B}$ is the basic image. $\Omega$ is the size of the filter window. $k$ is the normalization term:

$$
k=\sum_{i^{\prime}, j^{\prime} \in \Omega} \omega_{S}\left(\left\|i-i^{\prime}, j-j^{\prime}\right\|\right) \omega_{r}\left(\left\|I_{B}-I_{R}\right\|\right)
$$

The notation $i^{\prime}, j^{\prime} \in \Omega$ denotes that $\left(i^{\prime}, j^{\prime}\right)$ and $(i, j)$ are corresponding pixels from the adjacent frames. $\omega_{s}, \omega_{r}$ are two Gaussian kernels, in which $\omega_{s}$ is the kernel of spatial domain and $\omega_{r}$ is the kernel of intensity domain. The two Gaussian kernels could be expressed by the following equations:

$$
\begin{gathered}
\omega_{r}\left(I_{B}, I_{R}\right)=\exp \left(-\frac{\left\|I_{B}-I_{R}\right\|^{2}}{2 \sigma_{r}^{2}}\right) \\
\omega_{s}\left(\left(i, i^{\prime}\right),\left(j, j^{\prime}\right)\right)=\exp \left(-\frac{\left\|\left(i-i^{\prime}\right)-\left(j-j^{\prime}\right)\right\|^{2}}{2 \sigma_{s}^{2}}\right)
\end{gathered}
$$

Where $\sigma_{r}$ and $\sigma_{s}$ are the standard deviation of spatial and intensity. The two parameters define the extension of the two Gaussian kernels. $\sigma_{s}$ determines the size of the considered 
corresponding pixel pairs in the adjacent images and should be proportional to the image size. $\sigma_{r}$ determines the minimum amplitude of an edge. Since the adjacent images are very similar to each other, if the variation amplitude in the two images is less than $\sigma_{r}$, it will be smoothed by the JBF and the variation will leak into the detail layer. If the variation is sharper than $\sigma_{r}$, it will be less altered by the filter. The selection of these two parameters are very crucial for acquiring the best quality of the detail layer.

After the separation by the JBF, we can get the base layer and the detail layer noted as $I_{J B F}$ and $I_{d}$, respectively. $I_{R}$ is the reference image. The detail layer can be subtracted by the reference image and the base layer:

$$
I_{d}=I_{R}-I_{J B F}
$$

We process the two components separately. The base layer is projected into the proper range using modified histogram equalization technology raised in our previous research [23] and get the result $I_{J B F}^{\prime}$. Meanwhile, the detail layer is enhanced using the gain enhancement method and we get the result $I_{d}^{\prime}$. Finally, the two layers are recombined together for fully observation:

$$
I_{\text {out }}=I_{J B F}^{\prime}+I_{d}^{\prime}
$$

\subsection{Elimination of Gradient Reversal Artifacts}

In our previous research $[8,23]$, we found that the gradient reversal artifacts are caused by the non-linear filter such as the traditional bilateral filter. This effect is negligible when using the linear filter such as guided image filter. However, it cannot be fully eliminated by the guided image filter when strong edges on the image. When we use the JBF to separate the image, the gradient reversal artifacts are obvious because we use two adjacent images to calculate the detail layer. Although the adjacent images are quite similar to each other, there are still some tiny differences between them, especially in the strong edges and detail information. That is to say, the JBF can determine the edge and detail better than the traditional bilateral filter but somehow cause more gradient reversal artifacts. As we mentioned above, we can achieve better smooth effect by controlling the two Gaussian parameters. The more edge and detail information we get, the bigger odds that the gradient reversal artifacts will happen. We adopted an adaptive Gaussian filter in the previous research to eliminate the gradient reversal artifacts [8], but it is time-consuming for the algorithm. In this case, we design a new approach to eliminate the gradient reversal artifacts with a simple computational kernel function based on an adaptive emerge coefficient. This is greatly simplified the calculation process and get even better results.

The main problem when using the JBF to filter an image is that, it will cause the over-smooth effect on the edges or the slight detail information. Two aspects could explain the phenomenon: 1 . In the filter window of $\Omega$, when the intensity variation is too small in a 
spatial edge region, it is very hard to distinguish the weak edge from both the intensity domain and the spatial domain; 2. Even the intensity is discontinuousness in the corresponding spatial domain within the filter window $\Omega$, this consistency always has certain differences due to the quality of the imaging system. Similar pixel gray level exists in the spatial domain, especially within the edge region. We can see from eq. 1 and eq.2 that, the term $\mathrm{k}$ is determined by the parameter $\omega_{s}$ and $\omega_{r}$. When the dimension of the filter window $\Omega$ is determined, the effect of edge extraction is depended on $\omega_{r}$, but only this is not enough. It will cause the edge diffusion when the edge is too weak.

Zuo et al. [8] adopted a Gaussian filter to settle this problem, and progress had been accomplished. However, this approach could be time-consuming when applying the detail enhancement into real world hardware realization. Considering this, we have designed a new kernel function to deal with the weak edge and detail information. This kernel function is based on the thought of gradient constraint factor. We have found that, the gradient is more stable relative to the instability of the intensity. From the salient structure of image, we can say that, the gradient has more edge structure feature than the intensity variation. Thus, we give the expression of the newly designed kernel function for the JBF as eq.7 and eq.8:

$$
\begin{gathered}
f\left(i-i^{\prime}, j-j^{\prime}\right)=\omega_{s}\left(i-i^{\prime}, j-j^{\prime}\right) \omega_{e}\left(i-i^{\prime}, j-j^{\prime}\right) f_{k} \\
f_{k}=\alpha(\Omega) \omega_{r}\left(I_{B}-I_{R}\right)+(1-\alpha(\Omega)) \omega_{d}\left(i-i^{\prime}, j-j^{\prime}\right)
\end{gathered}
$$

Where the $f_{k}$ is the newly designed kernel function, $\omega_{e}$ is the gradient term, $\omega_{d}$ is the guided spatial similarity term, and $\alpha(\Omega)$ is the adaptive emerge coefficient.

Meanwhile, the guided spatial similarity term $\omega_{d}$ is expressed as:

$$
\omega_{d}\left(i-i^{\prime}, j-j^{\prime}\right)=\exp \left(-\frac{\left\|I_{B}\left(i-i^{\prime}, j-j^{\prime}\right)-I_{R}\left(i-i^{\prime}, j-j^{\prime}\right)\right\|}{2 \sigma_{d}^{2}}\right)^{2}
$$

The $\sigma_{d}$ is the standard deviation of the guided spatial similarity term. $\alpha(\Omega)$ is used to emerge the intensity similarity and the guided spatial similarity, which can be expressed as:

$$
\begin{aligned}
& \alpha(\Omega)=\frac{1}{\left(1+e^{-\Delta_{\Omega}}\right)} \\
& \Delta_{\Omega}=\frac{\left(\sigma_{d}+\varepsilon\right)}{\left(\sigma_{r}+\varepsilon\right)}
\end{aligned}
$$

$\varepsilon$ is the constraint factor, which could prevent the appearance of zero standard deviation. When the intensity variation is getting smaller while the spatial variation is getting bigger, the $\alpha(\Omega)$ is close to 1 , the intensity similarity is restrained. When the intensity variation is 
getting bigger while the spatial variation is getting smaller, the $\alpha(\Omega)$ is close to 0 , thus the spatial similarity is restrained. The gradient term $\omega_{e}$ is expressed as:

$$
\begin{gathered}
\omega_{e}\left(i-i^{\prime}, j-j^{\prime}\right)=\exp \left(-\frac{\left[\sum_{x, y} G_{x / y}\left(\nabla I_{B / R}, i-i^{\prime}, j-j^{\prime}\right)\right]^{2}}{2 \sigma_{e}^{2}}\right) \\
G_{x}\left(\nabla I_{B / R}, i-i^{\prime}, j-j^{\prime}\right)= \begin{cases}\sum_{h=i^{\prime}}^{i-1} \nabla I_{y}\left(h, i_{x}\right) & i_{x} \geq i_{X}^{\prime} \\
-\sum_{h=i^{\prime}}^{i-1} \nabla I_{y}\left(h, i_{x}\right) & \text { otherwise }\end{cases} \\
G_{y}\left(\nabla I_{B / R}, i-i^{\prime}, j-j^{\prime}\right)= \begin{cases}\sum_{h=j^{\prime}}^{j-1} \nabla I_{x}\left(j_{x}, h\right) & j_{y} \geq j_{y}^{\prime} \\
-\sum_{h=j^{\prime}}^{j-1} \nabla I_{x}\left(j_{x}, h\right) & \text { otherwise }\end{cases}
\end{gathered}
$$

In eq.13 and $14, \mathrm{x}$ and $\mathrm{y}$ represent the vertical and horizontal direction, respectively. $\nabla I_{x}$ and $\nabla I_{y}$ are the vertical and horizontal gradient respectively. $\sigma_{e}$ is the gradient standard deviation. We can see from these equations that $G_{X / y}$ represents the gradient variation level between the corresponding pixels in the adjacent images. We use two adjacent images to give a demonstration of the performance of the newly raise kernel function to show its effectiveness.

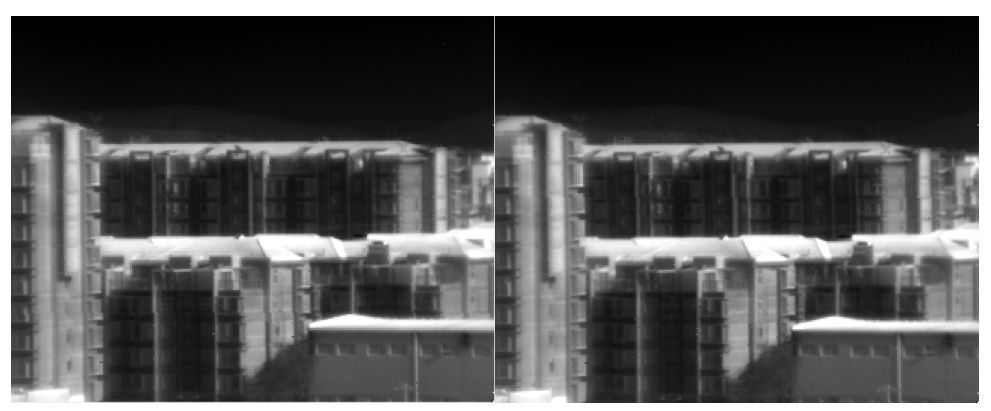

(a)

(b)

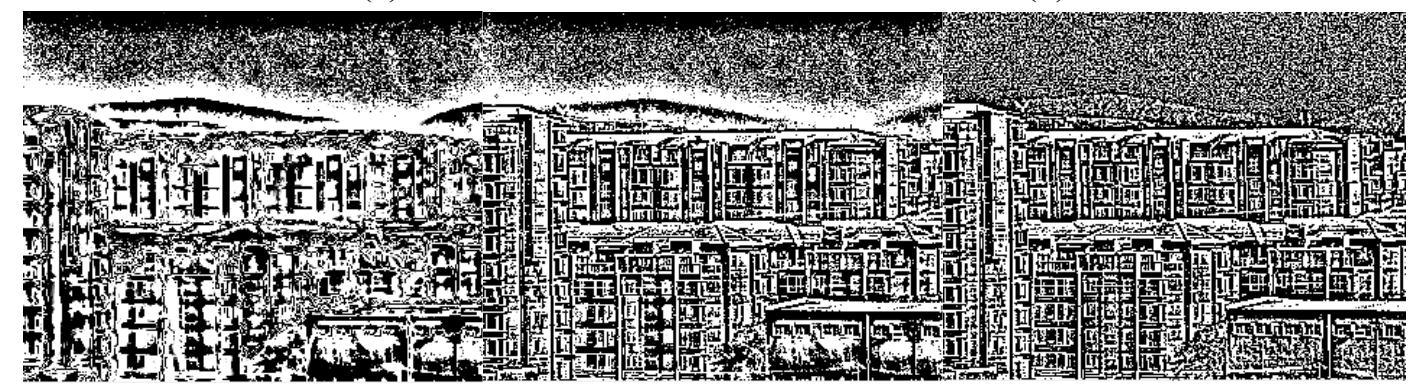

(c)

(d)

(e) 
Fig.2 performance comparison of gradient reversal artifacts elimination. (a) basic image; (b) reference image; (c) detail layer processed after the joint-bilateral filter; (d) gradient reversal artifacts removal using the Gaussian filter; (e) gradient reversal artifacts elimination using the newly raised kernel function.

Fig.2(a) and (b) are two adjacent images captured by a HgCdTe cooled infrared thermal imager with the sensitive wavelength of $3 \sim 5 \mu \mathrm{m}$. We use fig.2(a) as the basic image and fig.2(b) as the reference image. Fig.2(c) is the detail layer calculated by the joint-bilateral filter. As we can see in fig.2(c), the detail information is not very clear, the edges are somehow blurry. Fig.2(d) is the demonstration of fig.2(c) which is acquired by using the Gaussian filter [8]. Although the image has been greatly improved in visual effect, there are still some drawbacks. For example, in the lower-right corner, the detail information of the wall has become the shape of clusters. While in the upper region, the edge between the hill and the sky are ill processed. Meanwhile, the detail information in fig.2(d) seem unpleasant because they are not well-distributed enough. In fig.2(e), which is filtered by the newly raised kernel function, all the problem have been solved. In the lower-right corner, the wall has been clearly distinguished to what it ought to look like. The edge between the hill and sky is well outlined, and the detail information in the whole image are well-distributed. The visual effect of fig.2(e) is surely greatly improved compare to fig.2(d). According to the theoretical analysis above, the traditional bilateral filter and the joint-bilateral filter would both cause the problem of edge diffusion, which is extremely obvious at the edge of the hill and sky region. Although the Gaussian filter could somehow restrain the gradient reversal artifact, it could not prevent the edge diffusion according to its formula. The white overflow around this edge has clearly shown this problem. While according to our newly raised kernel function, we can greatly control the gradient information and restrain the edge diffusion as best as we can.

\subsection{Detail layer process technique}

According to fig.2(e), the current state of the detail layer cannot be used to conduct the reality reproduction of an infrared image. As we know, the noise information can be recognized as detail information when conducting the joint-bilateral filter and the new kernel function, and be filtered into the detail layer with the actual detail information. In fig.2(e), the sky region are occupied by noises which is not allow to happen when we finally reproduce and enhance the image.

Usually the noise is more sensitive according to the visual effect of an observer in flat regions. The human visual system is not keen to the noise in the complex background [14]. In the previous section, we have involved the adaptive emerge coefficient $\alpha(\Omega)$ to show which part of the image should be enhanced. Here we will approach how much each part should be enhanced. In a flat region, the value of $\alpha(\Omega)$ is near 0 . This coefficient will rise up when the fluctuation of pixels gets more obvious. Most of the values are under 1. If $\alpha(\Omega)$ exceed 0.95 , we assign them to 0.95 . Then, $I_{d}$ is derived from the following formula:

$$
I_{d}^{\prime}=I_{d} *(\alpha(\Omega) * a+b),
$$

Here $a$ and $b$ are the coefficients which set the gain to a suitable value. According to our 
research, we first set the value of $a=0.3$ and $b=0.65$ we can get a better result. Fig. 3 shows the effect of the gain adjustment for image Fig.2(e). We can see that, the noise in the flat sky region is starting to be suppressed. The choice of the coefficient $\mathrm{a}$ and $\mathrm{b}$ also affects the detail enhancing level of the building region. At the same time, the proper choice of the coefficient a and $b$ can suppress the noise. As mentioned above, in the flat region, the noise cannot be enhanced equal to the detail information. That means, since we use the coefficients of $a$ and $b$ in the whole detail layer, we have to evaluate their value to balance the noise suppression and the detail enhancement.

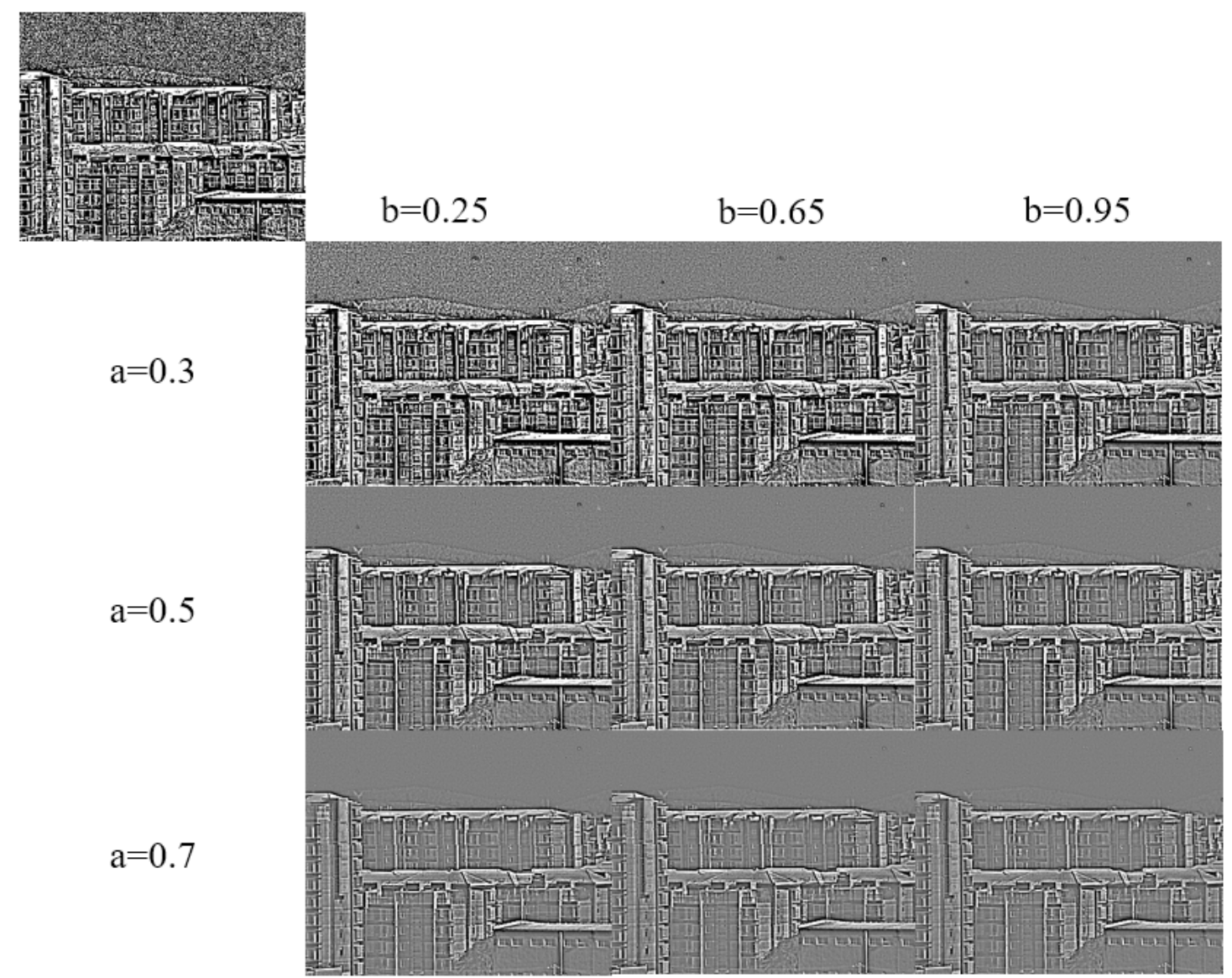

Fig. 3 the demonstration of gain controlling and noise suppression.

In fig.3, the coefficient $\mathrm{a}$ and $\mathrm{b}$ are set as different values to demonstrate their performance of gain controlling and noise suppression. We can see that, as the coefficient a and $\mathrm{b}$ go up, the noise in the sky region become lesser and lesser. However, the detail information in the building region become unclear as well. This could be an interaction between the noise suppression and the detail enhancement. When we applied this technique into real world application, we have to choose $\mathrm{a}$ and $\mathrm{b}$ carefully to suit the real scene situation. In our research, we choose $\mathrm{a}=0.5$ and $\mathrm{b}=0.65$ for the best visual display performance.

\section{Experimental Results}

In this section, we use several different sets of infrared images to compare our new approach with the proposed BF\&DDE and GIF\&DDE. The raw infrared images are captured by a $3 \sim 5 \mu \mathrm{m}$ HgCdTe cooled thermal imager with 16-bit output dynamic range. We focus on the subjective visual effect and the root-mean-square contrast (RMSC) index to compare the performance of the three methods. 


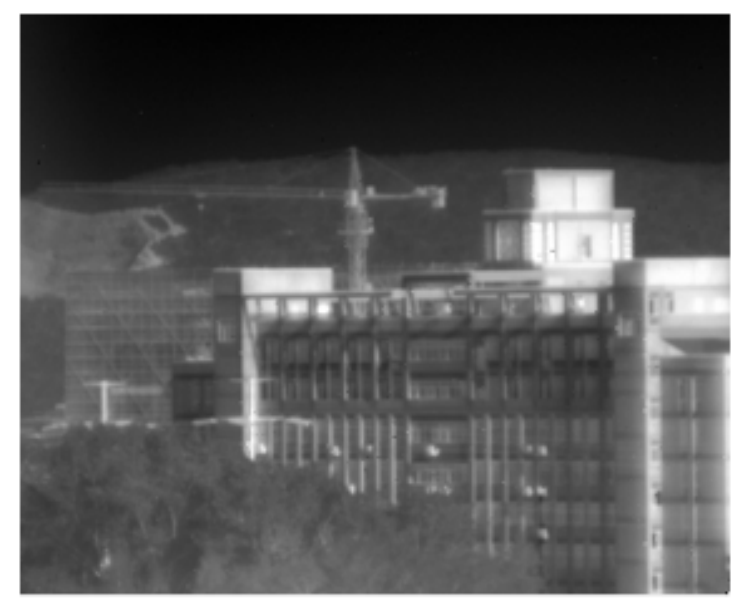

(a)

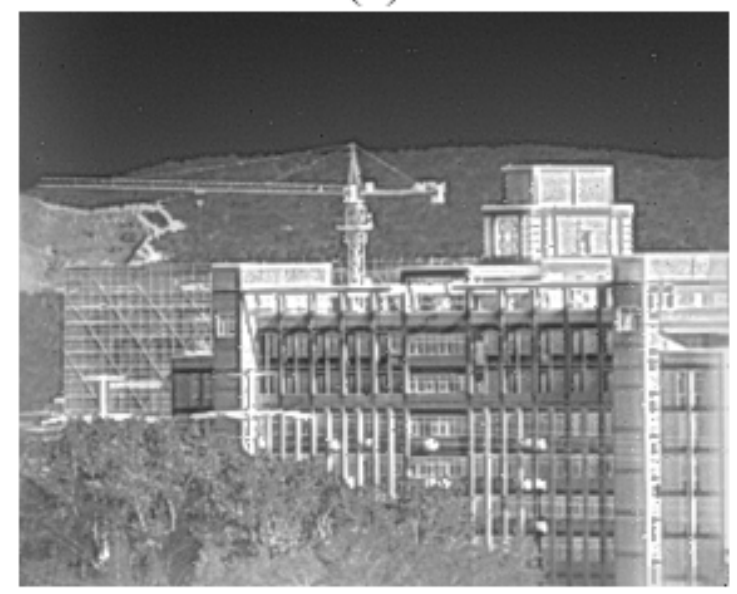

(c)

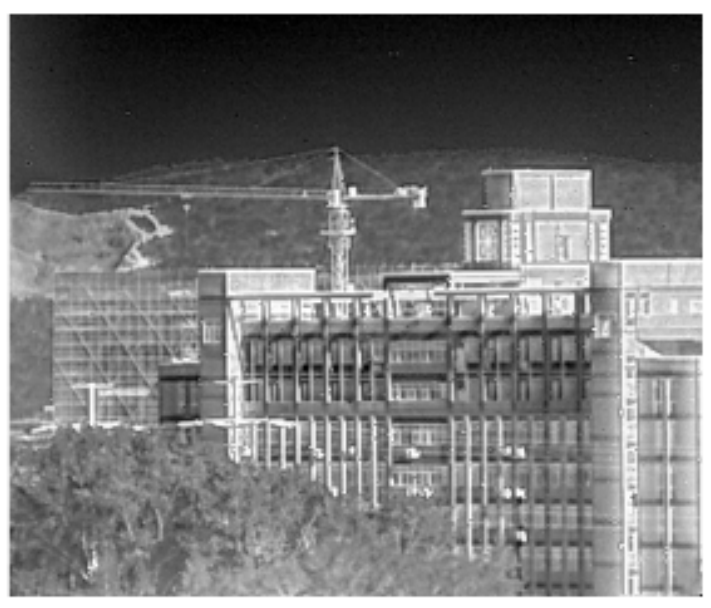

(b)

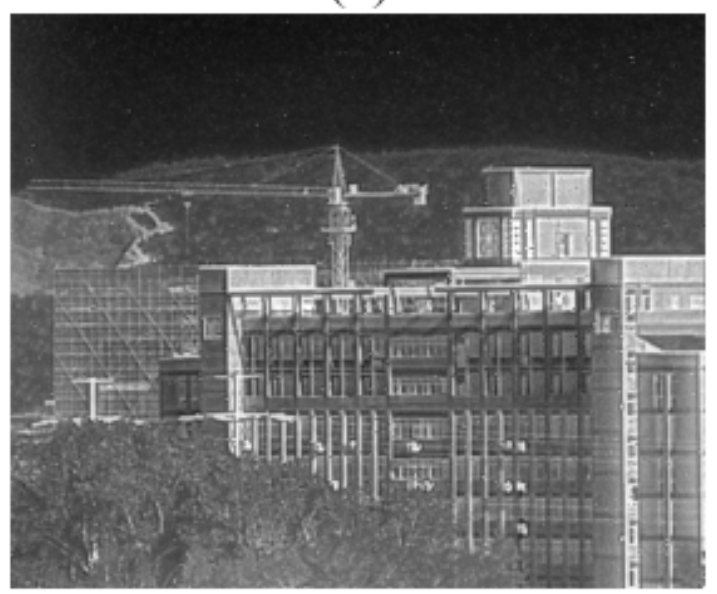

(d)

Fig.4 image contains trees, buildings, hill and sky. (a) the raw infrared image. (b)BF\&DDE. (c)GIF\&DDE. (d) our approach

First, we start with an infrared image contains complex information. In fig.4(a), there are trees, buildings, hill and sky in one scenario. All the three methods have enhanced the raw image into a whole new level. However, there are some different visual effect of them. The most obvious difference is the conjunction of the hill and the sky region. We can see a clear outline between the hill and sky region in fig.4(c) which is processed by the GIF\&DDE, resulting from that the GIF is a linear filter. However, it works less effective when dealing with the strong edges than the traditional BF and JBF due to its theoretical nature. Meanwhile, we can see a weak discontinuous outline in fig.2(b) with BF\&DDE, but in fig.4(d), we see nothing. According to the previous analysis, the Gaussian filter can somehow remove the gradient reversal artifact but become less effective when the edge is too strong. Our new kernel works better in eliminating the gradient reversal artifacts. Furthermore, when comparing the visual effect of these three processed images, both BF\&DDE and GIF\&DDE make the images too bright while our approach do not cause this problem. The scene looks more real with our approach. 


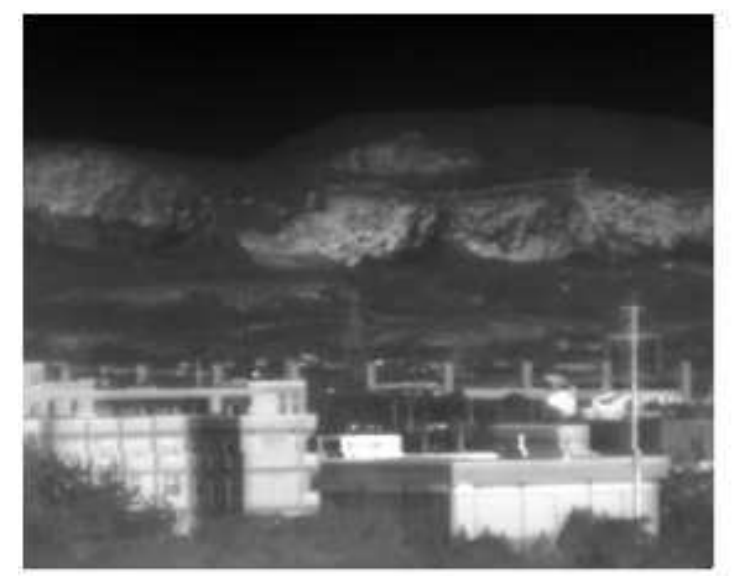

(a)

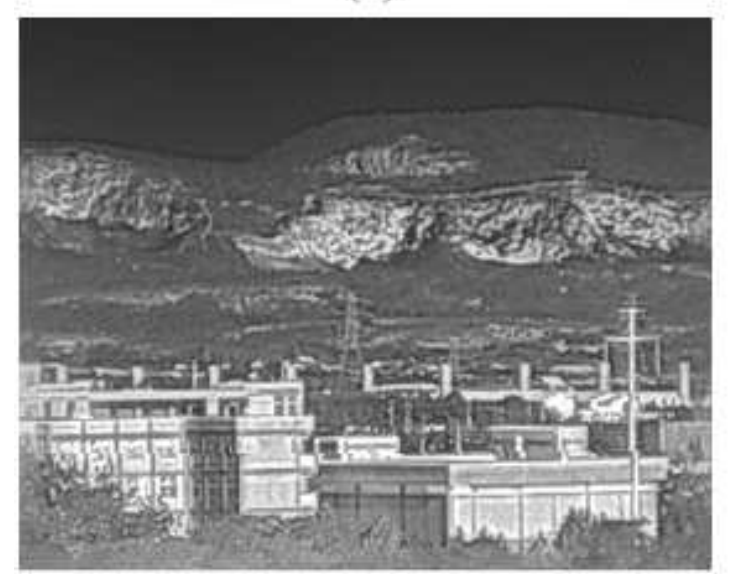

(c)

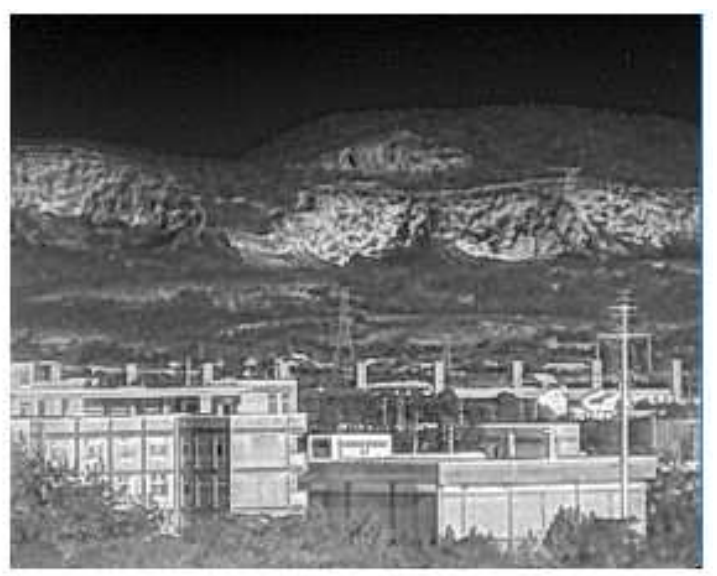

(b)

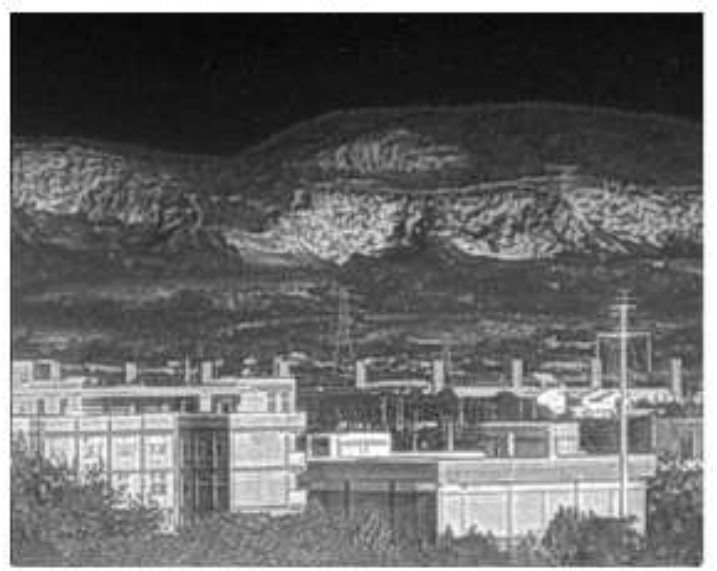

(d)

Fig.5 image contains more flat regions. (a) the raw infrared image. (b) BF\&DDE. (c) GIF\&DDE. (d) our approach

In fig.5, we choose an infrared image with more flat region to test the performance of these three techniques. The outline problem still appears here with the sequence of GIF\&DDE, BF\&DDE and our approach. Meanwhile, the BF\&DDE and GIF\&DDE have made the images too abrupt. This can be seen in the central region of the image. The visual effect of these two techniques is a little bit unreal. While in our approach, the image is smoother. In the lower region, the bushes look much clearer with our approach than the other two techniques. 


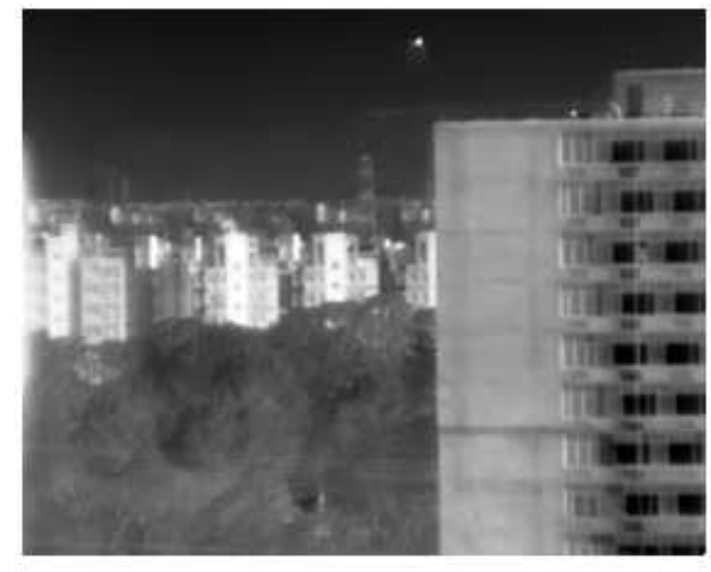

(a)

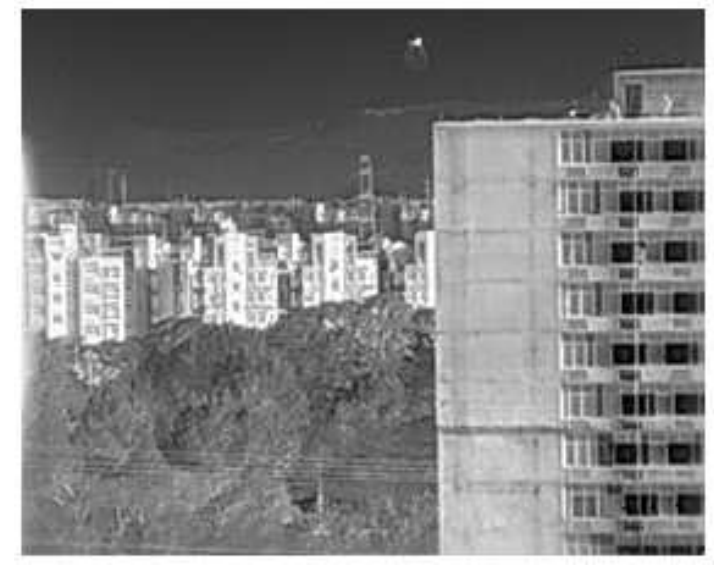

(c)

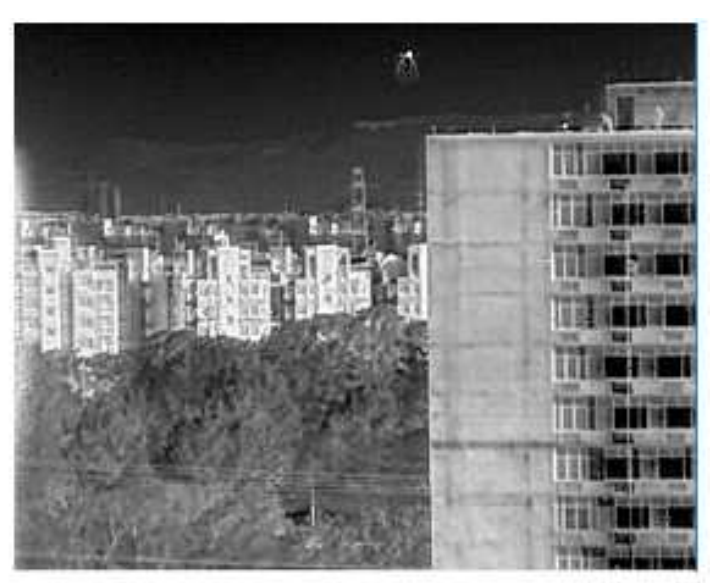

(b)

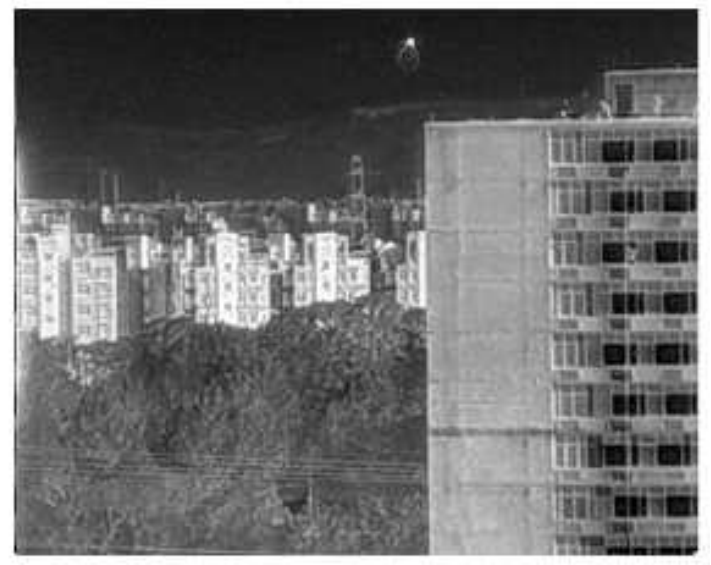

(d)

Fig.6 image contains more weak bushes information. (a) the raw infrared image. (b) BF\&DDE. (c)GIF\&DDE. (d) our approach

In fig.6, we focus on the visual effect on the large cluster of bushes. It is clearly that our approach works better than the other two techniques. The stems and leaves are well shaped in fig.4(d). The wall at the side of the building looks more like a real wall in fig.6(d) than in fig.6(b) and (c). The texture on the wall are reproduced better in fig.6(d). 


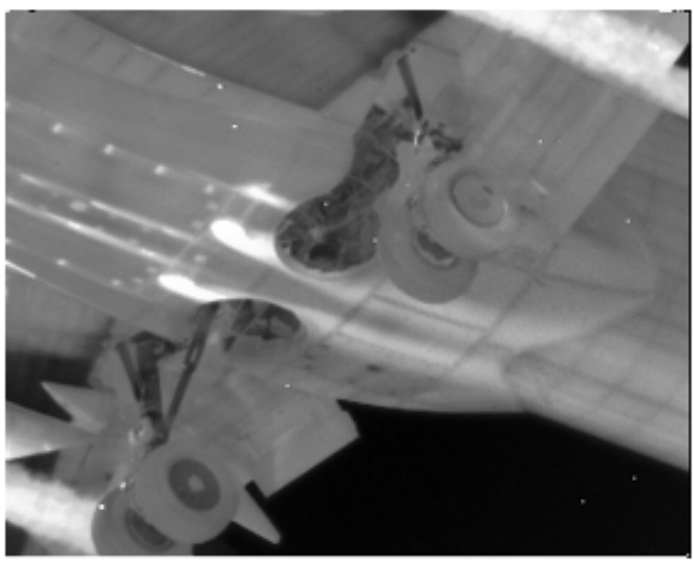

(a)

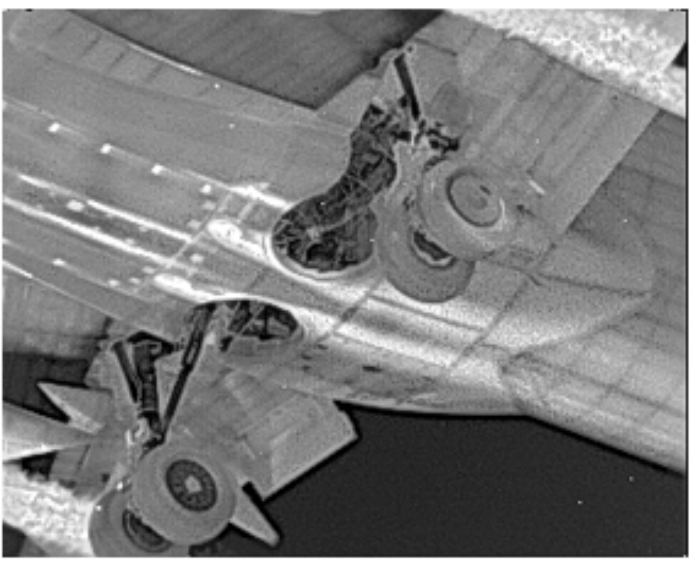

(c)

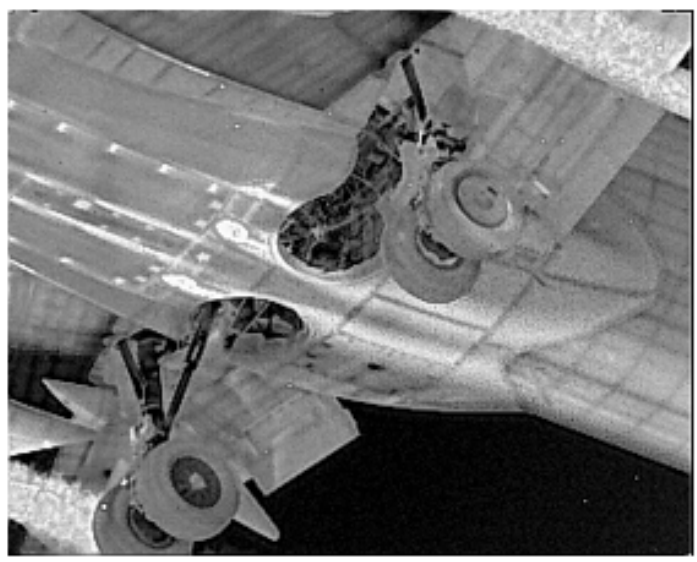

(b)

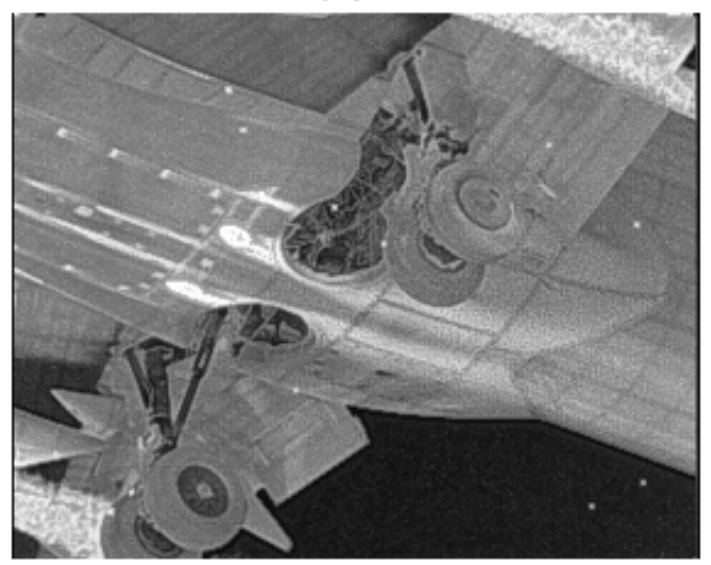

(d)

Fig.7 belly region of an airplane. (a) the raw infrared image. (b) BF\&DDE. (c) GIF\&DDE. (d) our approach

Fig.7 is a belly region of an airplane. Comparing the undercarriage retraction region, the mechanical structures are more obvious in fig.7(d) than in fig.7(b) and (c). The bright problem happens again in fig.7. Both fig.7(b) and (c) are unnatural brighter than fig.7(d). Our method not only enhances the detail information in the image but also maintains the bright and contrast level. This method reproduces the scene reality and makes the processed image watching like the real world appearance.

Next, we use the RMSC index to evaluate the performance of our new approach [8]. The RMSC index is used to quantitatively evaluate the enhancement effect of different methods, which is defined as:

$$
R M S C=\sqrt{\frac{1}{M \times N} \sum_{i, j}(I(i, j)-\bar{I})^{2}}
$$

where $\bar{I}$ is the average intensity of all pixel values in the image. $\mathrm{M}$ and $\mathrm{N}$ are the image's rows and columns, respectively. Note the RMSC is measured by calculating the average difference between the pixel values and the mean of the image. It reflects the amount of ambiguity in a picture that is measured, and larger values usually indicate better enhancement. The results of RMSC over each figure comparison are presented in fig.8. We calculate the 
RMSC as shown in fig.4-7 to demonstrate the superiority of our technology.

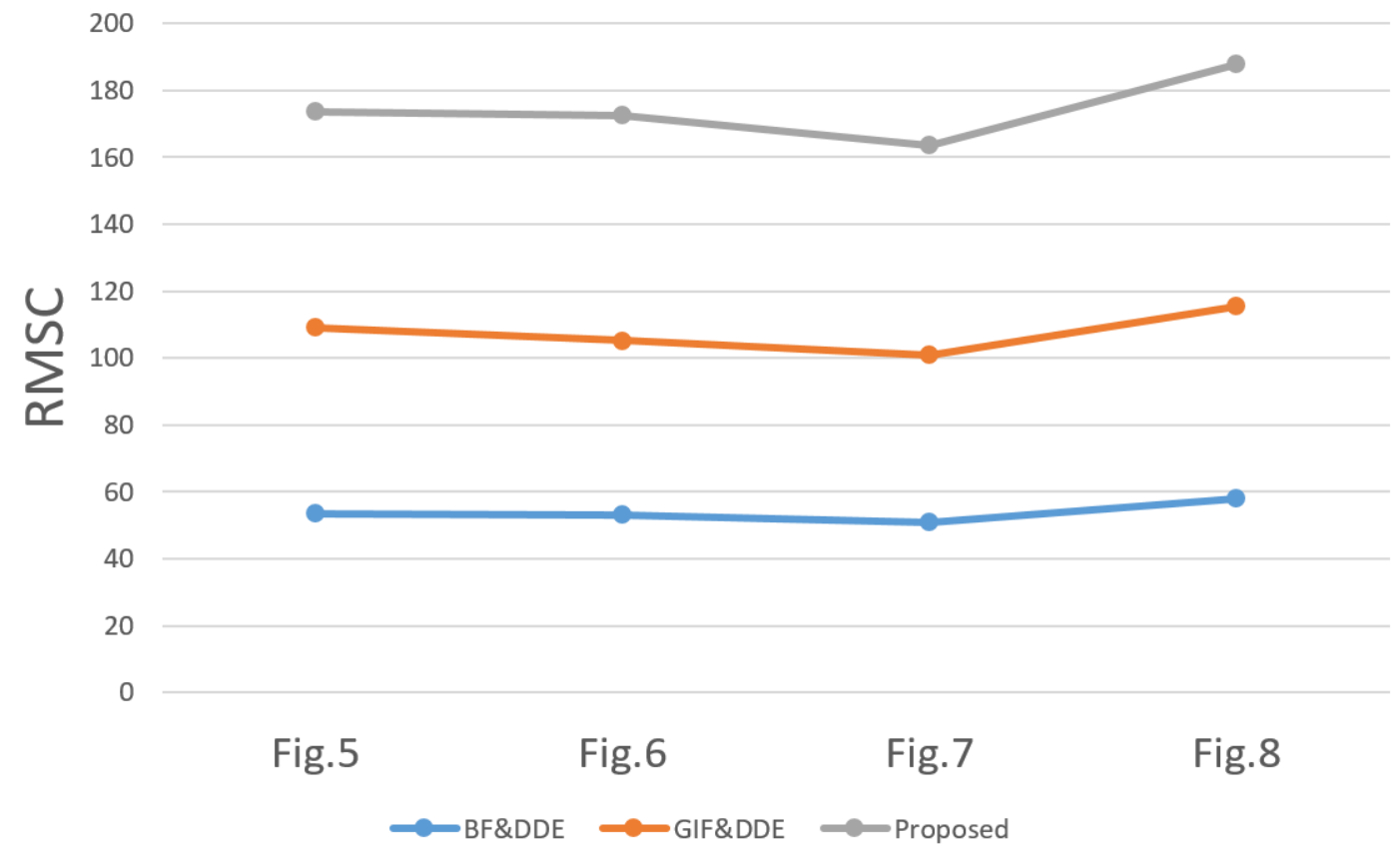

Fig.8 data charts of RMSC comparison of fig.5-fig.7. Gray dots and lines are the proposed method. Orange dots and lines are the GIF\&DDE. Blue dots and lines are the BF\&DDE.

Fig. 8 gives a clear demonstration of the RMSC index of these three methods. The numerical value shows that, our approach has the best numerical value. Here we give a simple explanation why the GIF\&DDE has the better numerical value than the BF\&DDE. According to eq.16, we find that the calculation of the RMSC may be affected by the noise level. Although we have analyzed that the GIF\&DDE may not work very effective as BF\&DDE, it works better in suppressing the noise than the BF\&DDE [8]. Meanwhile, our approach not only enhances the detail information, reproduces the reality of the image, and eliminates the gradient reversal artifacts but also suppresses the noise. Thus, it has the best numerical value of RMSC.

Meanwhile, we also use the background variation-detail variation(BV-DV) index to evaluate the results of these three methods. The BV-DV index has long be chosen as the evaluation criteria of image enhancement [20]. The principle of BV-DV is that, we separate the pixel gray intensity as "background pixel" and "detail pixel". As for one chosen pixel, if the gray intensity deviation between itself and the adjacent several pixels are small, this pixel is marked as background pixel, otherwise it is marked as detail pixel. After the enhancement process, the background gray intensity variation of the determined background pixels should be close to the BV calculated with the original raw image, while the detail gray intensity variation of the detail pixels should be bigger than the DV calculated with the original image. We use a $5 \times 5$ window to determine every pixel whether it should be belong to the background or the detail. After the determination, we calculate the BV of all the background pixels and the DV of all the detail pixels of fig.4-fig.7, respectively. Table 1 shows the BV-DV results of fig.4-fig.7.

Table 1 the BV-DV index of fig.4-fig.7

\section{Fig.4}

Fig.5

(a)

(c)

(d)

(a)

(b)

(c) 


\begin{tabular}{|c|c|c|c|c|c|c|c|c|}
\hline BV & 11.23 & 14.54 & 13.39 & 14.76 & 9.77 & 17.32 & 16.51 & 16.94 \\
\hline \multirow[t]{3}{*}{ DV } & 26.75 & 49.71 & 48.62 & 56.91 & 33.94 & 58.33 & 59.57 & 71.12 \\
\hline & \multicolumn{4}{|c|}{ Fig.6 } & \multicolumn{4}{|c|}{ Fig.7 } \\
\hline & (a) & (b) & (c) & (d) & (a) & (b) & (c) & (d) \\
\hline BV & 14.36 & 21.36 & 20.27 & 20.78 & 17.18 & 25.82 & 24.48 & 25.61 \\
\hline DV & 27.47 & 52.19 & 51.88 & 60.33 & 36.27 & 80.10 & 77.46 & 101.29 \\
\hline
\end{tabular}

Usually the value of BV should be less fluctuated before and after the enhancement, while the DV should be greatly improved. The bigger DV represents the better enhancement. As in table 1, the BV of all three methods are similar to the original value calculated with the original raw image, while the DV are greatly improved. We can see directly that, the proposed method has the biggest DV comparing to BF\&DDE and GIF DDE. This is another demonstration of that our method could enhance the image better.

\section{Conclusion}

In this paper, we focus on how to enhance the detail within the raw infrared image with a new approach, and proposed a novel technology to accomplish this goal. This technology is based on the improved joint-bilateral filter, and we design a new kernel function to eliminate the gradient reversal artifacts, distinguish the detail information from the noise and finally enhance the infrared image. This technology has so far the better visual effect and the numerical value than the previous research. We are still working on how to simplify the computational process in order to bring this technology out of the laboratory into the real-world industrial application. We are sure that this technology will give great help in many military and civil fields.

\section{Acknowledgement}

This research was supported by the National Science Foundation of China under Grant No. 61505083; The introduction of talent school scientific research fund under Grant No. NY215043.

\section{References}

[1] J. Silverman, Display and enhancement of infrared images, in International Conference on Image Processing and its Applications, pp. 345-348, 1992.

[2] R. C. Gonzalez and R. E. Woods, Digital Image Processing (2nd Edition), Prentice Hall, New Jersey, 2003.

[3] S. M. Pizer, E. P. Amburm, J. D. Austin, R. Cromartie, A. Geselowitz, T. Greer, B. T . H. Romeny, and J. B. Zimmerman, Adaptive histogram equalization and its variations, Comput. Vis. Graph. Image Process, 39(3), pp. 355-368, 1987.

[4] K. Zuiderveld, Contrast limited adaptive histogram equalizaiton, in Graphics Gems IV, pp. 474-485, Academic Press Professional, Inc., San Diego , 1994.

[5] J. Kim, L. Kim, and S. Hawng, IEEE Trans. Circ. Syst. Video Technol. 11, 475, 2001.

[6] F. Branchitta, M. Diani, G. Corsini, and A. Porta, Dynamic-rang compression and contrast enhancement in infrared imaging system, Opt. Eng. 47(7), 076401, 2008.

[7] F. Branchitta, M. Diani, G. Corsini, and A. Porta, New technique for the visualization of high dynamic range infrared images, Opt. Eng. 48(9), 096401, 2009.

[8] C. Zuo, Q. Chen, N. Liu, Display and detail enhancement for high-dynamic-range 
infrared images, Opt. Eng. 50(12), 127401, 2011.

[9] K. He, J. Sun, X. Tang, Guided image filtering, ECCV 2010, Part I, LNCS 6311, pp. 1-14, 2010.

[10] R. Lai, Y. T. Yang, B. J. Wang and H. X. Zhou, A quantitative measure based infrared image enhancement algorithm using plateau histogram, Opt Commun 283(21), pp. 4283-4288, 2010.

[11] C. C. Pham, S. V. U. Ha, J. W. Jeon, Adaptive guided image filtering for sharpness enhancement and noise reduction, PSIVT 2011, Part I, LNCS 7087, pp. 323-334, 2011.

[12] A. Goyal, A. Bijalwan, P. Kumar, Image enhancement using guided image filter technique, JIEAT, Vol. 1, pp. 213-217, 2012.

[13] V. E. Vickers, Plateau equalization algorithm for real-time display of high-quality infrared imagery, Opt. Eng. 35(1921 ), 1996.

[14] G. L. Anderson and A. N. Netravali, Image restoration based on a subjective criterion, IEEE Trans. Syst. Man Cybern. 6(12), pp. 845-853, 1976.

[15] B. Liu, X. Wang, W. Jin, Y. Chen, Infrared image detail enhancement based on local adaptive gamma correction, Chin Opt. Lett 10(2), 021002, 2012.

[16] C. L. Lin, C. L., An approach to adaptive infrared image enhancement for long-range surveillance, Infrared Phys. Tech 54(2), pp. 84-91, 2011.

[17] J. Zhou, R. Dai, B. Xiao, Overview of image quality assessment research, Comp. Sci. 7(35), 2008.

[18] E. Peli, Contrast in complex images, J. Opt. Soc. Am. A 7(10), pp. 2032-2040, 1990.

[19] H Shin, T Yu, Y Ismail, B Saeed, "Rendering high dynamic range images by using integrated global and local processing." Optical Engineering 50.11: 117002-117002,2011.

[20] A Rossi, N Acito, M Diani, G Corsini, "Dynamic range reduction and contrast adjustment of infrared images in surveillance scenarios." Optical Engineering 52.10: 102002-102002,2013.

[21]C. Zuo, Q. Chen, X. Sui, "Range Limited Bi-Histogram Equalization for image contrast enhancement", Optik-International Journal for Light and Electron Optics 124(5), 425-431,2013.

[22] C. Zuo, Q. Chen, X. Sui, and J. Ren, "Brightness preserving image contrast enhancement using spatially weighted histogram equalization", IAJIT 50, 4468,2014.

[23]N. Liu, D. X. Zhao, "Detail enhancement for high-dynamic-range infrared images based on guided image filter", Infrared Phys. Tech 67:138-147, 2014.

[24]J. Kopf, M. F. Cohen, D. Lischinski, M. Uyttendaele, "Joint Bilater Upsampling", ACM Transactions on Graphics, 26(3), 96:1-6, 2007. 